\title{
Human Milk to Prevent and Heal Gastrointestinal Tract Injury in Children After Bone Marrow Transplantation
}

\author{
Stella M. Davies
}

B ONE MARROW TRANSPLANTATION is a long, difficult treatment associated with many complications. An intentional, lethal dose of chemotherapy or total body radiation is administered to replace unhealthy bone marrow with new bone marrow stem cells. This can result in damage to other organs (particularly the gut), bacteremia, and graft-versushost disease (GVHD). In GVHD, injury to epithelial cells causes loss of epithelial barrier function and subsequent production of proinflammatory cytokines that trigger the activation of donor-derived T cells. Acute GVHD can affect the skin, eyes, gut (colon and upper gastrointestinal tract), and liver. Severe GVHD of the gut results in infection, especially from gut organisms getting into the blood, and is the main reason why children die after bone marrow transplant.

Recent data suggest that there is a progressive loss of gut microbiome diversity, particularly Clostridiales and other obligate anaerobic bacteria, that is associated with use of antibiotic therapy after bone marrow transplant. ${ }^{1}$ A study in 42 children undergoing transplant found that luminal (i.e. stool) short chain fatty acid (SCFA) levels progressively declined within 2 weeks after transplant. A reduction in luminal butyrate levels correlated with a loss of butyrate, producing bacteria in the gut microbiota. Lower SCFA levels after bone marrow transplant may allow for increases in formate and diminished hypoxia in the colonic epithelium, promoting the expansion of Enterobacteriaceae, which may contribute to the development of GVHD. In support of this, reduced levels of luminal SCFAs were observed in children with GVHD after transplant suggesting a direct link between GVHD and the gut microbiota.

Enteral human milk given after transplant could be used to modify the microbiome and reduce the incidence of GVHD in children after bone marrow transplant. Results from a small pilot study in children aged 6 to 40 months demonstrated that donor milk was well tolerated and associated with a reduction in levels of soluble interleukin (IL)-2 receptor (sIL-2r), an inflammatory marker. In partnership with Prolacta, a larger randomized trial was conducted in children $<5$ years of age undergoing transplant $(N=28)$. Patients received either donor milk or their mothers' own milk $(n=24)$ or received conventional nutritional support $(n=4)$. The incidence of GVHD was significantly lower in patients who received human milk than in controls (Table 1); the incidence of bacteremia was also significantly lower in patients who received human milk $(12.5 \%$ versus $50 \% ; p=0.14)$.

Levels of proinflammatory cytokines (i.e., IL-6, IL-10, IL8 , interferon- $\gamma[\mathrm{IFN} \gamma]$, and sIL-2r) were lower at day 14 after transplant in patients who received human milk than in controls. Preliminary analyses of bacterial diversity showed higher species richness and greater abundance of Streptococcus and Lactobacillus in stool samples from patients who received human milk. Thus, the initial findings from this study suggest that children receiving enteral human milk may have lower levels of inflammatory cytokines in the blood and maintain a more diverse microbiome after transplant than children not receiving human milk. However, these results must be interpreted with caution due to the limited sample size.

In summary, disruption of intestinal homeostasis and the microbiome is a major cause of morbidity and mortality (e.g., bacteremia and GVHD) after bone marrow transplantation. Preliminary data suggest that it is feasible to administer human milk to young children in this setting, and this intervention might reduce inflammation and GVHD. Further studies are needed to determine the effects of donor

Table 1. Graft-Versus-Host Disease in Allogeneic Bone Marrow Transplant

\begin{tabular}{|c|c|c|c|}
\hline & Received human breast milk $(\mathrm{n}=23)$ & Controls $(\mathrm{n}=4)$ & $p$ \\
\hline Any GVHD & $17 \%(n=4)$ & $75 \%(n=3)$ & 0.04 \\
\hline GI GVHD & $13 \%(n=3)$ & $50 \%(n=2)$ & 0.14 \\
\hline Grades 2-4 GVHD & $9 \%(n=2)$ & $50 \%(n=2)$ & 0.09 \\
\hline
\end{tabular}

GI, gastrointestinal; GVHD, graft-versus-host disease.

Jacob G. Schmidlapp Endowed Chair and Professor of Pediatrics, and Director of Bone Marrow Transplantation and Immune Deficiency, Cincinnati Children's Hospital Medical Center, Cincinnati, Ohio. 
human milk on gut microbiota in children undergoing bone marrow transplant.

\section{Reference}

1. Jenq RR, Ubeda $\mathrm{C}$, Taur $\mathrm{Y}$, et al. Regulation of intestinal inflammation by microbiota following allogeneic bone marrow transplantation. J Exp Med 2012;209:903-911.
Address correspondence to: Stella M. Davies, MBBS, PhD, MRCP Cincinnati Children's Hospital Medical Center 3333 Burnet Avenue Cincinnati, $\mathrm{OH} 45229$

E-mail: stella.davies@cchmc.org 